\title{
Erratum to: Mutational landscape of mucinous ovarian carcinoma and its neoplastic precursors
}

Georgina L. Ryland', Sally M. Hunter ${ }^{1}$, Maria A. Doyle ${ }^{2}$, Franco Caramia², Jason Li², Simone M. Rowley ${ }^{1}$, Michael Christie ${ }^{3}$, Prue E. Allan ${ }^{4}$, Andrew N. Stephens ${ }^{5,6,7}$, David D. L. Bowtell ${ }^{8,9,10}$, Australian Ovarian Cancer Study Group, lan G. Campbell ${ }^{1,9,11}$ and Kylie L. Gorringe ${ }^{1,9,11^{*}}$

\section{Erratum}

It has come to our attention that there is an error in Additional file 2 for this article [1]. When opening Additional file 2, this links to the incorrect file. The correct version of Additional file 2 can be found below. The publishers apologise for any inconvenience caused.

\section{Reference}

1. Ryland GL, Hunter SM, Doyle MA, Caramia F, Li J, Rowley SM, Christie M, Allan PE, Stephens AN, Bowtell DDL, Australian Ovarian Cancer Study Group, Campbell IG, Gorringe KL. Mutational landscape of mucinous ovarian carcinoma and its neoplastic precursors. Genome Med. 2015;7:87.

\section{Additional file}

Additional file 2: Figure S1. Nucleotide substitution frequency and context. Figure S2. RRAS2 somatic mutation. Figure S3. Genetic comparison between mucinous ovarian tumors and mucinous cancers from other anatomical sites. Figure S4. ELF3 somatic mutations. Figure S5. H\&E stained sections of frozen tissues used for exome discovery cohort. (PDF 9813 kb)

\begin{abstract}
Author details
${ }^{1}$ Cancer Genetics Laboratory, Peter MacCallum Cancer Centre, East Melbourne, VIC, Australia. ${ }^{2}$ Bioinformatics Core Facility, Peter MacCallum Cancer Centre, East Melbourne, VIC, Australia. ${ }^{3}$ Department of Anatomical Pathology, Royal Melbourne Hospital, Parkville, VIC, Australia. ${ }^{4}$ Department of Pathology, Peter MacCallum Cancer Centre, East Melbourne, VIC, Australia. ${ }^{5}$ Centre for Cancer Research, MIMR-PHI Institute of Medical Research, Clayton, VIC, Australia. ${ }^{6}$ Department of Molecular and Translational Sciences, Monash University, Clayton, VIC, Australia. ${ }^{7}$ Epworth Research Institute, Epworth HealthCare, Richmond, VIC, Australia. ${ }^{8}$ Cancer Genetics and Genomics Laboratory, Peter MacCallum Cancer Centre, East Melbourne, VIC, Australia. ${ }^{9}$ Sir Peter MacCallum Department of Oncology, University of Melbourne, Parkville, VIC, Australia. ${ }^{10}$ Department of Biochemistry and Molecular Biology, University of Melbourne, Parkville, VIC, Australia. ${ }^{11}$ Department of Pathology, University of Melbourne, Parkville, VIC, Australia.
\end{abstract}

Received: 15 December 2016 Accepted: 15 December 2016 Published online: 12 January 2017

\footnotetext{
* Correspondence: kylie.gorringe@petermac.org

${ }^{1}$ Cancer Genetics Laboratory, Peter MacCallum Cancer Centre, East Melbourne, VIC, Australia

${ }^{9}$ Sir Peter MacCallum Department of Oncology, University of Melbourne, Parkville, VIC, Australia
} 